\title{
An improved optical method for surface plasmon resonance experiments
}

\author{
A. T. M. Lenferink*, R .P. H. Kooyman and J. Greve \\ Department of Applied Physics, Twente University, P.O. Box 217, 7500 AE Enschede (The Netherlands)
}

(Received October 11, 1990; accepted in revised form May 23, 1991)

\begin{abstract}
In this paper an inexpensive optical device is described, which is capable of measuring the optical reflectance at different angles, while keeping the laser spot stationary at one point of the surface. This is accomplished by applying cylindrical optics. Its use is demonstrated in a surface plasmon resonance (SPR) sensor. A coil-operated vibrating mirror is used to obtain an angle scan of about 4 degrees. The angle of minimum reflectance can be detected with an accuracy of approximately $2 \times 10^{-3}$ degrees. Despite the use of simple optical components, disturbance of laser beam parallelism is no more than 0.02 degrees. Displacement of the laser spot at the surface during the angle scan is kept within $0.2 \mathrm{~mm}$. The device eliminates disturbances due to surface irregularities in measurements.
\end{abstract}

\section{Introduction}

Surface plasmon resonance (SPR) occurs when light passing through a medium (e.g., glass) with a certain refractive index reflects at a thin metal layer (e.g., silver or gold of about $53 \mathrm{~nm}$ ), which is in contact with a medium of lower refractive index (e.g., buffer solution). At a certain angle $\left(\theta_{\mathrm{pl}}\right)$ a sharp minimum in reflectance is found [1]. SPR is used as a sensor, as the angle $\theta_{\mathrm{pl}}$ strongly depends on the refractive index of the medium on top of the metal layer. The high sensitivity in the immediate vicinity $(\approx 100 \mathrm{~nm})$ of the metal layer makes it very useful in research on thin layers [2, 3]. An example is found in immunosensor applications, where antibodies are immobilized on the surface in order to monitor immunoreactions [4-9].

The most commonly used method for SPR measurements is a set-up with a prism mounted on a rotation stage, where rotation of the stage leads to the different angles inside the prism. This method, however, is slow and has a relatively low angular resolution ( 0.01 degrees). This type of set-up is mostly used in fixed-angle measurements [4, $5,7]$. A shift in SPR minimum angle $\left(\theta_{\mathrm{pl}}\right)$

*Author to whom correspondence should be addressed. will then be measured as a change in reflected intensity. Another approach is to apply a focused light beam to the metal interface and to detect the angular-dependent reflectance using a diode array $[8,10,11]$. In particular, the Pharmacia biosensor group has been very active in developing a practical SPR sensor system based on this optical read-out principle $[8,12]$. Here, the shift in SPR angle due to a change in refractive index profile near the metal-solution interface is calculated by fitting a suitably chosen mathematical function to the relative intensities measured by $\approx 100$ pixels in the diode array. In their instrument, a shift of the location of the minimum of one pixel unit corresponds to a refractive index change of approximately $10^{-3}$ [8]. For low concentrations of analyte, the net SPR response can be as low as $10^{-5}$ refractive index units, and thus one has to be very confident in the fitting procedure to be able to interpret the experimental data for these low concentrations. Further, the time response of the instrument is $\approx 100 \mathrm{~ms}$, which is mainly determined by the maximum attainable sampling rate. This time constant can be too high for those cases where one is interested in the determination of surface reaction rate constants. Recently, we de- 


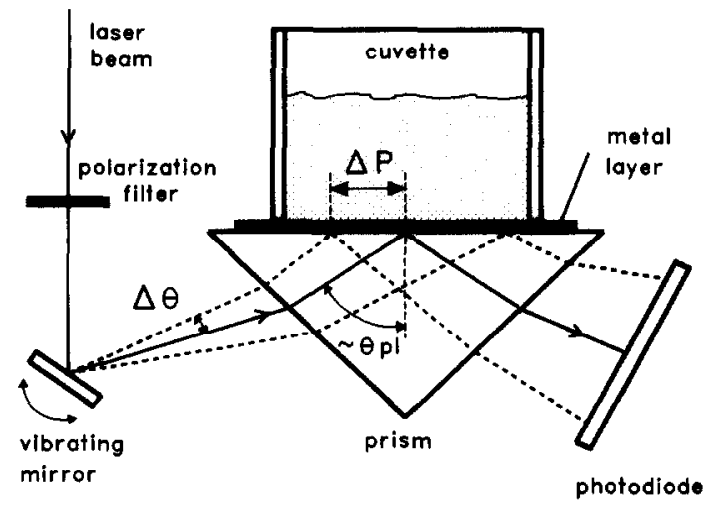

Fig. 1. Set-up for SPR measurements using a vibrating mirror. A change in angle $(\Delta \theta)$ causes a displacement $(\Delta P)$ of the laser beam at the metal surface.

scribed an inexpensive SPR device which does not suffer from these limitations. In this setup [9] the angular shift is actually measured with a resolution of approximately $10^{-3} \mathrm{de}-$ grees, corresponding to a refractive index resolution of $\approx 10^{-5}$, without relying on fitting procedures. Furthermore, by using a mirror vibrating at $f \approx 50 \mathrm{~Hz}$ an angular range of $\approx 4$ degrees is scanned in $20 \mathrm{~ms}$, which determines the time constant of the system. It is a simple matter to decrease this time constant by increasing the vibration frequency of the mirror. However, a disadvantage of this set-up is the displacement $(\Delta P)$ of the laser beam at the metal surface during the angle $\operatorname{scan}(\Delta \theta)$ (see Fig. 1). In our SPR measurements an additional polystyrene layer is often used to improve the adsorption of antibodies to the surface. This polystyrene layer is made by spin casting, which produces $210 \AA$ layers with a thickness homogeneity of $5 \%$. Therefore different positions on the surface correspond to different plasmon angles $\left(\theta_{\mathrm{pl}}\right)$. This effect will lead to deformation of the measured SPR curve during the angle scan. It is possible, however, to keep the laser beam fixed at one point of the metal surface during the angle scan by choosing a different optical layout. In this way the plasmon angle is measured at one position and artifacts due to spatial inhomogeneities are eliminated.

\section{The device}

A schematic drawing of the new device is shown in Fig. 2. The prism from Fig. 1 is

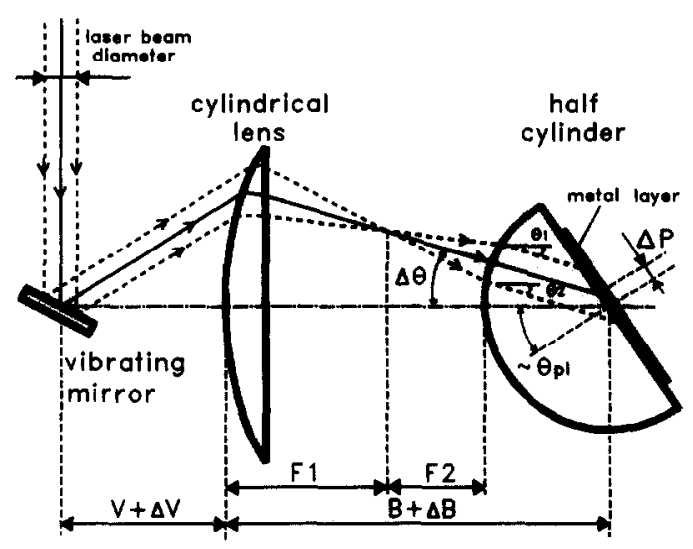

Fig. 2. Principle of the new set-up for SPR measurements. Some cylindrical optics are used to eliminate the displacement $(\Delta P)$ at the surface when scanning different angles $(\Delta \theta)$.

replaced by a half cylinder. The optics in our set-up are used to keep a parallel light beam inside the half cylinder, and scan all angles with the coil-operated mirror. The function of the cylindrical lens is two-fold. It projects the rotating axis of the mirror at the centre of the half cylinder and compensates its converging effect. If the distances $V$ and $B$ (see Fig. 2) are chosen properly, distortion of the laser beam inside the half cylinder $\left(\theta_{1}-\theta_{2}\right)$ and displacement of the laser beam over the metal surface $(\Delta P)$ will be kept small for different values of $\Delta \theta$. Since it is impossible to keep both $\theta_{1}-\theta_{2}=0$ and $\Delta P=0$ for all possible angles $\Delta \theta$, a ray-trace program (applying Snell's law) is used to determine the optimum values for $V$ and $B$ for a commonly used SPR angular amplitude $\left(\Delta \theta_{\max }\right)$ of approximately 1.8 degrees. The results from these calculations are presented in Figs. 3 and 4. These Figures show the results both for the optimized and non-optimized situations. In the non-optimized situation, $V$ and $B$ are determined by the paraxial design equations:

$B=F_{1}+F_{2}+R_{2}$

and

$\frac{1}{V}+\frac{1}{B}=\frac{1}{F_{1}}$

with $F_{i}$ the focal length of lens $i$ and $R_{i}$ the radius of lens $i$. $V$ represents the distance between the mirror and the cylindrical lens 


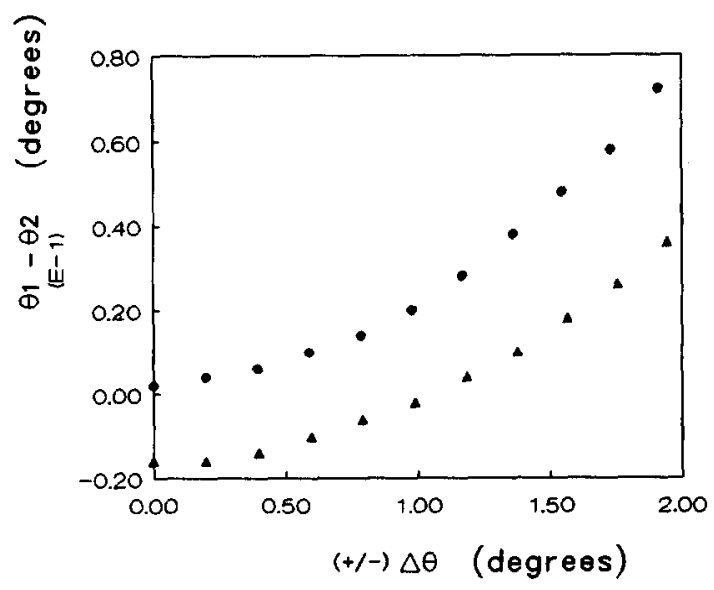

Fig. 3. Beam divergence caused by the optics used in the new set-up, for the optimized (A) and non-optimized situations (O).

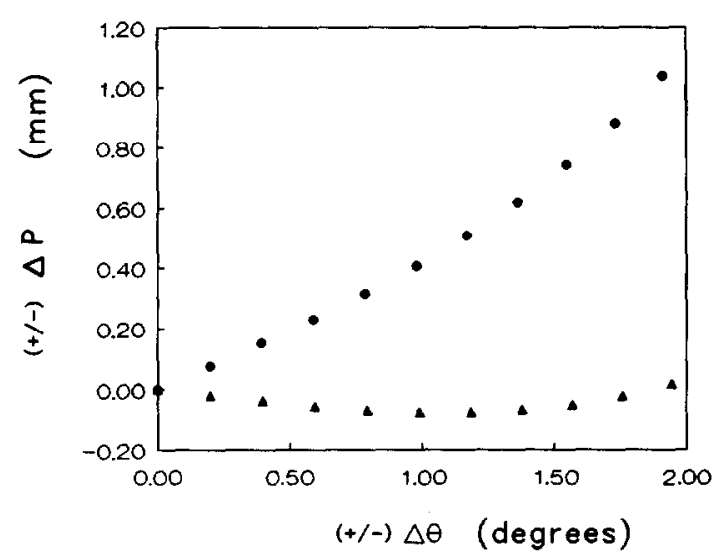

Fig. 4. Beam displacement $(\Delta P)$ at the metal surface at different angles $(\Delta \theta)$ for the optimized $(\Delta)$ and nonoptimized situations (O).

(object distance) and $B$ represents the distance between the cylindrical lens and the metal layer (image distance). In the optimized situation some small changes are made to the distances by adding $\Delta V$ and $\Delta B$ to obtain the best results for an angular amplitude $\left(\Delta \theta_{\max }\right)$ of 1.8 degrees. Figure 3 shows that the beam divergence during the angle scan is kept within 0.02 degrees and Fig. 4 shows that the displacement of the laser beam at the metal surface is less than $0.2 \mathrm{~mm}$. These are very acceptable values for SPR measurements if one takes into account the diameter of the laser spot, which is approximately $1 \mathrm{~mm}$. Using the set-up from Fig. 1 a displacement $\triangle P$ of about $13 \mathrm{~mm}$ would have been found under the same angular scan conditions. The absence of beam displacement becomes important when additional layers with inhomogeneous thickness variations are used on top of the metal layer. Different plasmon minimum angles then appear at different positions on the surface. Using the set-up from Fig. 1, this will lead to considerable distortion of the SPR measurements. However, the new set-up will still give a correct SPR signal for all angles.

\section{Results}

To demonstrate the potential of the new device, both set-ups from Figs. 1 and 2 are compared under the same conditions. To record the SPR curve, an a.c. current is sent to the mirror. For not too large amplitudes

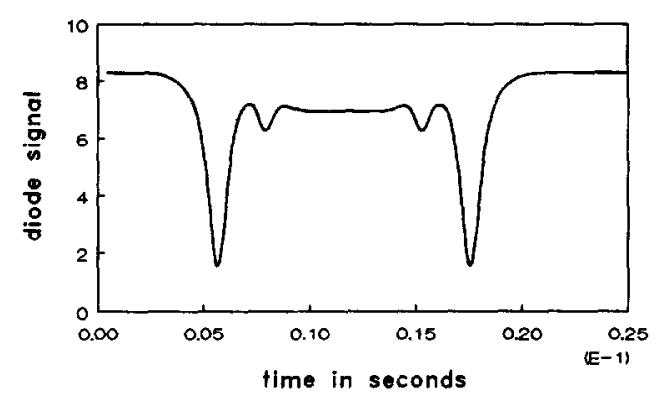

Fig. 5. SPR curve measured with the 'old' set-up, using a $530 \AA$ silver layer with an additional spin-coated polystyrene layer $(210 \AA)$ in air. The light source was a 632.8 $\mathrm{nm} \mathrm{He}-\mathrm{Ne}$ laser. During one cycle $\left(44 \mathrm{~Hz}, 1.87^{\circ}\right)$ of the mirror, the SPR minimum is detected twice. Disturbance of the SPR curve is caused by surface irregularities and dispacement of the laser beam at the metal surface during the angle scan.

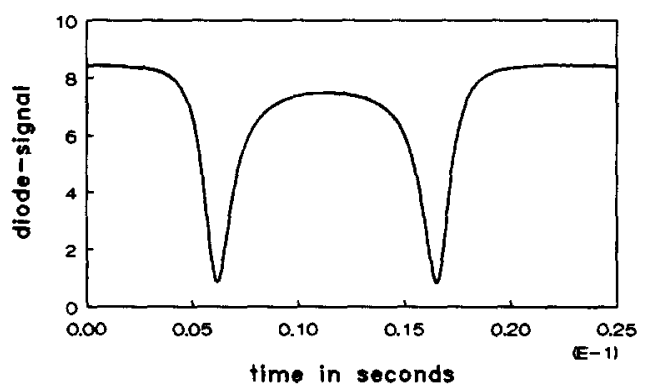

Fig. 6. SPR curve measured with the new set-up (same conditions as Fig. 5). Due to the absence of laser-beam displacement during the angle scan, no distortion of the SPR curve is seen here, despite the presence of surface irregularities. 
(maximum 5 degrees), the angle is proportional to the current, so $\Delta \theta=\Delta \theta_{\max }$ $\sin \left(\omega t+\phi_{0}\right)$ [9]. In our measurements we used a $\Delta \theta_{\max }$ of 1.87 degrees, and a frequency of $44 \mathrm{~Hz}$. During one cycle of the vibrating mirror, the beam traverses the reflectance minimum twice. The light intensity is measurement by a large-area photodiode (BPY63P). The light-intensity signal is stored on disk by an XT-microcomputer (equipped with an RTI-800 interface board). A glass microscope slide is used on which the metal layer (in this case $530 \AA$ of silver) is evaporated. Also a spin-coated polystyrene layer about $210 \AA$ thick is added on the metal surface. This disposable part of the device is optically coupled to the prism or half cylinder with some matching oil. For measurements of Figs. 5 and 6 the same glass slide is used in both set-ups. In Fig. 5 the reflectance as a function of time, measured using the set-up as shown in Fig. 1, shows a deformed SPR curve due to the thickness inhomogeneity of the polystyrene layer and/or surface flatness variations of the glass slide. Figure 6 shows the result obtained with the new device. No deformation of the SPR curve is seen here, because the same position on the surface is measured during the angle scan.

\section{Discussion}

We have demonstrated the improvement of an already existing vibrating-mirror angle scan set-up for SPR use. The elimination of the displacement of the laser beam during the angular scan makes the device insensitive to surface flatness variations, so cheap disposable glass microscope slides may be used for each SPR measurement. The new device is also insensitive to thickness variations of additional layers added on the metal surface, because the probed spot remains essentially stationary during the angle scan. Here it should be mentioned that this improvement is of course of less relevance when very small changes in SPR angles are measured. An additional advantage of the 'one point angle scan' is that plasmon minimum angles in the range 80-89 degrees (appearing when using a gold layer with polystyrene and buffer me- dium) are still easy to measure. In the setup used formerly this is only possible if the angular amplitude is decreased considerably.

\section{References}

1 H. Raether, in G. Hass, M. Francombe and R. Hoffman (cds.), Physics of Thin Films, Vol. 9, Academic Press, New York, 1977, pp. 145-261.

2 J. D. Pollard, G. W. Bradberry and J. R. Sambles, A study of the thin metal film/fluid interface using surface plasmon-polaritons, Solid State Commun., 63 (1987) 803-806.

3 J. Giergiel, C. E. Reed, S. Ushioda and J. C. Hemminger, ATR study of pyridine overlayers on silver films, Phys. Rev. B, 31 (1985) 3323-3329.

4 B. Liedberg, C. Nylander and I. Lundström, SPR for gas detection and biosensing, Sensor and Actuators, 4 (1983) 299-304.

5 M. T. Flanagan and R. H. Pantell, SPR and immunosensors, Electron. Lett., 20 (1984) 968-969.

6 D. C. Cullen, R. G. W. Brown and C. R. Lowe, Detection of immuno-complex formation via SPR on gold-coated diffraction gratings, Biosensors, 3 (1988) 211-225.

7 R. P. H. Kooyman, H. Kolkman, J. van Gent and J. Greve, Surface plasmon resonance immunosensors: sensitivity considerations, Anal Chim, Acta, 213 (1988) $35-45$.

8 B. Ivarsson, U. Jönsson, R. Karlsson, B. Liedberg, B. Renck, H. Roos, II. Sjödin, E. Stenberg, R. Ståhlberg, C. Urbaniczky and I. Lundström, Bioanalysis with surface plasmon resonance: principles, Proc. 3rd Int. Meet. on Chemical Sensors, Sept. 24-26, 1990, pp. 157-160.

9 R. P. H. Kooyman, A. T. M. Lenferink, R. G. Eenink and J. Greve, A vibrating mirror surface plasmon resonance immunosensor, Anal. Chem., 63 (1991) 83-85.

10 M. F. Finlan, S. A. Charles, J. C. Irlam and J. E. M. Midgley, European Patent No. EP 0305109 Al (Aug. 18, 1988).

11 K. Matsubara, S. Kawata and S. Minami, Optical chemical sensor based on surface plasmon measurement, Appl. Opt., 27 (1988) 1160-1163.

12 BLAcore Methods Manual, Pharmacia Biosensor, AB Uppsala, Sweden, 1990.

\section{Biographies}

Aufried Lenferink received the Ing. degree in technical physics from the Hogere Technische School Enschede (The Netherlands) in 1984. From 1984 to 1985 he worked as a physicist at Twente University in the low temperature group, and from 1985 to 1987 as process engineer at STORK Boilers. Since 1987 he has been working at Twente University as a physicist, supporting the biophysics technology group. 
Rob Kooyman received his Ph.D. in molecular biophysics from the Agricultural University in Wageningen (The Netherlands) in 1980. Since then he has been involved in biophysical membrane research. He has been working at Twente University in the Applied Physics Department since 1985, where his main interests are the development of optical immunosensors and characterization of biointerfaces.
Jan Greve received his Ph.D. in physics from the Free University in Amsterdam (The Netherlands) in 1972. Since then he has conducted research in molecular and cellular biophysics. In 1980 he was appointed as a full professor in the Applied Physics Department of the University of Twente, where he is now head of the biophysics technology group. 